\title{
Sensitive Spectroscopic Detection of Large and Denatured Protein Aggregates in Solution by Use of the Fluorescent Dye Nile Red
}

\author{
Marc Sutter • Sabrina Oliveira • Niek N. Sanders • Bart Lucas • Arie van Hoek • \\ Mark A. Hink • Antonie J. W. G. Visser • Stefaan C. De Smedt • Wim E. Hennink • \\ Wim Jiskoot
}

Received: 20 October 2006 / Accepted: 2 January 2007 / Published online: 9 February 2007

(C) Springer Science+Business Media, LLC 2007

\begin{abstract}
The fluorescent dye Nile red was used as a probe for the sensitive detection of large, denatured aggregates of the model protein $\beta$-galactosidase (E. coli) in solution. Aggregates were formed by irreversible heat denaturation of $\beta$ galactosidase below and above the protein's unfolding temperature of $57.4^{\circ} \mathrm{C}$, and the presence of aggregates in heated solutions was confirmed by static light scattering. Interaction of Nile red with $\beta$-galactosidase aggregates led to a shift of the emission maximum $\left(\lambda_{\max }\right)$ from 660 to $611 \mathrm{~nm}$, and to an increase of fluorescence intensity. Time-resolved fluorescence and fluorescence correlation spectroscopy (FCS) measurements showed that Nile red detected large aggregates with hydrodynamic radii around $130 \mathrm{~nm}$. By steadystate fluorescence measurements, it was possible to detect $1 \mathrm{nM}$ of denatured and aggregated $\beta$-galactosidase in solution. The comparison with size exclusion chromatography (SEC) showed that native $\beta$-galactosidase and small aggregates thereof had no substantial effect on the fluorescence of Nile red. Large aggregates were not detected by SEC, be-
\end{abstract}

M. Sutter $(\bowtie) \cdot$ W. Jiskoot

Division of Drug Delivery Technology, Leiden/Amsterdam Center

for Drug Research, Leiden University,

P.O. Box 9502, 2300 RA Leiden, The Netherlands

e-mail: m.sutter@lacdr.leidenuniv.nl

S. Oliveira · W. E. Hennink · W. Jiskoot

Department of Pharmaceutical Sciences, Faculty of Science,

Utrecht Institute for Pharmaceutical Sciences (UIPS), Utrecht

University,

Sorbonnelaan 16,

3584 CA Utrecht, The Netherlands

N. N. Sanders · B. Lucas · S. C. De Smedt

Laboratory of General Biochemistry and Physical Pharmacy,

Faculty of Pharmaceutical Sciences, Ghent University,

Harelbekestraat 72,

9000 Ghent, Belgium cause they were excluded from the column. The results with $\beta$-galactosidase demonstrate the potential of Nile red for developing complementary analytical methods that overcome the size limitations of SEC, and can detect the formation of large protein aggregates at early stages.

Keywords Protein aggregation · Fluorescence spectroscopy $\cdot$ Fluorescence correlation spectroscopy $\cdot$ Nile red $\cdot$ Size exclusion chromatography

\section{Introduction}

Among the biological macromolecules, proteins have become an important source of active substances for medical purposes. Insulin and growth hormone are two prominent examples of the many proteins used in medicine nowadays [1]. Many protein formulations can cause immune reactions

A. van Hoek

Laboratory of Biophysics, MicroSpectroscopy Centre,

Wageningen University,

Dreijenlaan 3,

6703 HA Wageningen, The Netherlands

M. A. Hink · A. J. W. G. Visser

Laboratory of Biochemistry, MicroSpectroscopy Centre,

Wageningen University,

Dreijenlaan 3,

6703 HA Wageningen, The Netherlands

\section{A. J. W. G. Visser}

Department of Structural Biology, Faculty of Earth and Life

Sciences, Vrije Universiteit,

De Boelelaan 1087,

1081 HV Amsterdam, The Netherlands 
in patients, and the presence of protein aggregates, even in minute amounts, has been identified as an important factor leading to the breaking of immune tolerance $[2,3]$. Large protein aggregates are regarded as particularly immunogenic [4], and for this reason, analytical procedures for their sensitive and selective detection are important. The most widely used technique for analyzing aggregates in protein formulations is size exclusion chromatography (SEC) [2]. However, large protein aggregates of more than about 5 million $\mathrm{Da}$ are out of the separation range of SEC and can even be excluded from separation columns. Moreover, proteincolumn interactions may cause aberrant elution leading to erroneous interpretations. Field flow fractionation and analytical ultracentrifugation have a greater capacity than SEC for detecting high molecular weight protein aggregates [5, 6], but until now, these techniques are not readily available in every laboratory. Analysis by light scattering techniques [7] and Fourier transform infrared spectroscopy (FTIR) [8-10] is experimentally easy in the sense that these techniques do not rely on physical separation steps. However, the former is prone to artifacts when aggregates are present at low concentrations, and the latter is not very sensitive. Fluorescence spectroscopy with probes covalently labeled to proteins has been used for the direct detection of aggregated species [11], but this approach suffers from the problem that the modified protein may exhibit an altered aggregation behavior. Methods without protein labeling are rarer, but environment sensitive fluorescent dyes can be useful for detecting aggregated structures. For example, Congo red is used for the detection and identification of amyloid fibrils [12, 13]. Another potentially interesting dye is Nile red, which is established for the detection of hydrophobic areas on the surfaces of proteins [14-17]. Recently, this dye has been used for the detection of aggregates of an IgG1 recombinant humanized monoclonal antibody, as well as human calcitonin fibrils by fluorescence microscopy [18].

The fluorescence of Nile red is strongly dependent on the polarity of its environment. Decreasing polarity leads to a substantial blue shift of the absorption and emission maxima, as well as a marked increase of quantum yield and fluorescence lifetime. This has been related to the large dipole moment of the molecules in the excited state and the existence of a non-emissive twisted intramolecular charge transfer (TICT) state, which is more likely to be formed in polar than apolar environments [19, 20]. It has been proposed that the TICT state is non-emissive because of fast, non-radiative transition to the triplet state [19]. Nile red is a hydrophobic molecule with low solubility in water of $\sim 1 \mu \mathrm{M}$ [21]. Therefore, it has the tendency to partition into hydrophobic phases [22].

$\beta$-Galactosidase from Escherichia coli is used as a model protein in the present study. This tetrameric enzyme $(\mathrm{MW}=465 \mathrm{kDa})$ consists of four equal subunits. It con- tains four catalytic sites for the hydrolysis of lactose or other $\beta$-galactosides to monosaccharides. For maximal enzymatic activity, the presence of $\mathrm{Mg}^{2+}$ or, alternatively, $\mathrm{Mn}^{2+}$ is required [23, 24]. It has been shown that $\mathrm{Mg}^{2+}$ increases the unfolding temperature of $\beta$-galactosidase in aqueous solution. In the presence of $\mathrm{Mg}^{2+}$, heat induced unfolding is irreversible. It coincides with activity loss and leads to the formation of denatured monomers, dimers, and aggregates [25].

In this study, steady-state and time-resolved fluorescence spectroscopy experiments with Nile red are used for the sensitive detection of large $\beta$-galactosidase aggregates, present in solution at concentrations in the low nanomolar range. Denaturation and aggregation of native protein is induced by incubating aqueous solutions below and above the experimentally determined unfolding temperature of $57.4^{\circ} \mathrm{C}$. The presence of aggregates in heat-treated solutions is confirmed by light scattering. Time-resolved fluorescence anisotropy and fluorescence correlation spectroscopy (FCS) are used for analyzing the size of the aggregates detected by Nile red. The results obtained with Nile red are compared with results from SEC analysis.

\section{Materials and methods}

\section{Materials}

Freeze-dried $\beta$-Galactosidase from Escherichia coli (protein content $\sim 80 \%$ ) was purchased from Fluka (Buchs, Switzerland) and stored at $-20^{\circ} \mathrm{C}$. Phosphate buffer ( $\mathrm{pH}$ 7.4) containing $55 \mathrm{mM}$ sodium-phosphate and $10 \mathrm{mM} \mathrm{MgCl} 2$ was prepared with reverse osmosis water, and rendered isotonic by the addition of $\mathrm{NaCl}$. Upon preparation, buffer solution was filtered through a $0.2 \mu \mathrm{m}$ cellulose acetate filter (Schleicher and Schuell, Dassel, Germany), and stored at $4^{\circ} \mathrm{C}$ for at most 4 weeks. $0.2 \mu \mathrm{m}$ HPLC filters were obtained from Alltech (Deerfield, IL). Nile red (9-diethylamino-5Hbenzo $[\alpha]$ phenoxazine-5-one) was obtained from Fluka. A stock solution containing $0.25 \mathrm{mM}$ Nile red was prepared with water-free dimethylsulfoxide (DMSO) and stored at $-20^{\circ} \mathrm{C}$.

\section{Methods}

\section{Preparation of $\beta$-galactosidase solution}

A fresh solution of $\beta$-galactosidase in phosphate buffer was prepared before each experiment. The protein was dissolved in the buffer and subsequently, the solution was filtered through a $0.2 \mu \mathrm{m}$ HPLC filter. Protein concentration after filtration was determined by measuring absorption at $280 \mathrm{~nm}$, 
assuming an absorption coefficient of $2.09 \mathrm{~cm}^{2} / \mathrm{mg}$ [26]. Reproducibly, a protein concentration close to $0.10 \mu \mathrm{M}$ was found after filtration.

For incubation at elevated temperatures, $1 \mathrm{ml}$ aliquots of $\beta$-galactosidase solution containing $0.10 \mu \mathrm{M}$ protein were filled into $1.5 \mathrm{ml}$ polypropylene Eppendorf tubes. They were heated for a defined period of time without shaking using an Eppendorf Thermomixer (Eppendorf, Hamburg, Germany), and then cooled at room temperature.

Steady-state fluorescence measurements

\section{Instrument and measurement settings}

Steady-state fluorescence measurements were performed with a Fluorolog FL3-21 spectrofluorometer (Jobin Yvon Horiba, Edison, NJ), equipped with a short-arc xenon lamp. The slit openings of the excitation and emission monochromators were set to a bandwidth of $3 \mathrm{~nm}$. The integration time was $0.05 \mathrm{~s}$, and the signals were corrected for lamp intensity fluctuations by a simultaneously recorded reference signal. Each sample was measured 3-5 times, and the average spectrum was calculated. The sample temperature was controlled by a water bath with a temperature sensor connected to the sample holder. Samples were measured in quartz cuvettes (Hellma GmbH, Muellheim, Germany).

\section{Intrinsic tryptophan fluorescence}

Measurements of intrinsic tryptophan fluorescence of $\beta$ galactosidase were performed by exciting $0.10 \mu \mathrm{M}$ protein solutions at $298 \mathrm{~nm}$ and scanning emission between 310 and $450 \mathrm{~nm}$. To establish a protein denaturation curve, the sample temperature was increased from 25 to $70^{\circ} \mathrm{C}$ in one degree steps. At every temperature, the sample was equilibrated for 5 min and then measured.

\section{Nile red fluorescence}

Before every measurement, a fresh solution of $25 \mu \mathrm{M}$ dye in phosphate buffer was prepared by dilution of Nile red stock solution. This solution was immediately added to heated $0.10 \mu \mathrm{M} \beta$-galactosidase solution, non-heated $0.10 \mu \mathrm{M}$ $\beta$-galactosidase solution or phosphate buffer in a cuvette, and mixed well. The final concentration of Nile red was $18.75 \mathrm{nM}$, well below the solubility limit of Nile red in water of $1 \mu \mathrm{M}$ [21]. Nile red fluorescence was measured by exciting at $550 \mathrm{~nm}$ and scanning emission between 565 and $750 \mathrm{~nm}$. All measurements were performed at $25^{\circ} \mathrm{C}$. Fluorescence spectra were measured immediately after addition of Nile red. However, Nile red emission in water remained unchanged for at least 25 min upon sample preparation.
Time-resolved fluorescence measurements

\section{Instrument and measurement settings}

Time-resolved fluorescence measurements were performed by time-correlated single photon counting, as described in detail elsewhere [27]. Excitation source was a mode-locked titanium:sapphire laser (model Verdi V10, Coherent Inc., Santa Clara, CA), pumped by a CW diode-pumped, frequency doubled Nd: $\mathrm{YVO}_{4}$ laser (model Mira $900-\mathrm{D}$ in fs mode, Coherent Inc., Santa Clara, CA), and tuned at $980 \mathrm{~nm}$. An excitation wavelength of $490 \mathrm{~nm}$ was obtained by frequency doubling, using a second harmonic generation system (model 5-050 Ultrafast Harmonic Generation System, Inrad Inc., Northvale, NJ). The repetition rate of the excitation pulses was $3.8 \mathrm{MHz}$. The emission filters were a Schott $3 \mathrm{~mm}$ OG $530 \mathrm{~nm}$ cut-off filter (Schott AG, Mainz, Germany), combined with a Schott IL $611.6 \mathrm{~nm}(\Delta \lambda=11.0 \mathrm{~nm})$ interference filter.

Measurements were performed at $25^{\circ} \mathrm{C}$, and consisted of repeated $10 \mathrm{~s}$ sequences of measuring parallel and perpendicularly polarized fluorescence emission until a maximum peak content of at least 50,000 counts in the data files was reached. Samples of $\beta$-galactosidase solution without Nile red were measured for background correction. To minimize background luminescence, all solutions, including buffer, were prepared with fluorescence spectroscopy grade water (Fluka, Buchs, Switzerland). For the performance of a deconvolution procedure in data analysis, the dynamic instrumental response of the experimental setup was recorded using the fast and single-exponential fluorescence decay of the reference compound erythrosine B in water.

Data analysis was performed using the "TRFA Data Processing Package" of the Scientific Software Technologies Center (Belarusian State University, Minsk, Belarus) [28]. The decay of fluorescence intensity with time, $I(t)$, could be described by an exponential model with three independent fluorescence lifetimes $\left(\tau_{i}\right)[29,30]$ :

$I(t)=\sum_{i=1}^{3} \alpha_{i} \cdot \exp \left(-t / \tau_{i}\right)$

where $\alpha_{i}$ are the pre-exponential factors, which were normalized. $I(t)$ itself was calculated from the measured parallel and perpendicular polarized fluorescence emission, $I_{\mathrm{II}}(t)$ and $I_{\perp}(t)$ :

$I(t)=I_{\mathrm{II}}(t)+2 \cdot G \cdot I_{\perp}(t)$

$G$ in the above equation represents the $g$-factor, which had a value of 1 for the used experimental setup. 
The course of fluorescence anisotropy with time $r(t)$ was calculated from the measured intensities $I_{\mathrm{II}}(t)$ and $I_{\perp}(t)$ :

$r(t)=\frac{I_{\mathrm{II}}(t)-G \cdot I_{\perp}(t)}{I_{\mathrm{II}}(t)+2 G \cdot I_{\perp}(t)}$

Fluorescence correlation spectroscopy measurements

\section{Instrument and measurement settings}

Fluorescence correlation spectroscopy measurements were performed with a system composed of a krypton-argon laser and an MRC1024 confocal laser-scanning microscope (Biorad, Hercules, CA), a TE300D inverted microscope (Nikon, Tokyo, Japan) with a water immersion objective lens (Plan Apo 60×, NA 1.2, collar rim correction, Nikon), and ALV5000/E avalanche photodiode detectors (ALV GmbH, Langen, Germany). The $568 \mathrm{~nm}$ line of the krypton-argon laser was used for excitation of Nile red samples. Fluorescence emission above $585 \mathrm{~nm}$ was detected.

Samples were prepared by mixing heated $0.10 \mu \mathrm{M} \beta$ galactosidase solution, non-heated $0.10 \mu \mathrm{M} \beta$-galactosidase solution or phosphate buffer with diluted Nile red stock solution in polypropylene Eppendorf tubes. Heated $\beta$ galactosidase solution was mixed with Nile red in the same tubes that were used for heating. The final concentration of Nile red in the samples was $18.75 \mathrm{nM}$. One hundred $\mu \mathrm{l}$ sample was transferred into a well of a 96 glass bottom well plate (Bio-one, Greiner, Frickenhausen, Germany). For measurements, the focal volume was positioned $100 \mu \mathrm{m}$ above the bottom of the well. Raw data, i.e. fluctuations of fluorescence intensity with time, were collected during $30 \mathrm{~s}$. The laser power was minimized to prevent photobleaching during this period. Every sample was measured ten times, and ten experimental autocorrelation functions were subsequently obtained from the raw data.

Analysis of the experimental autocorrelation functions was performed by non-linear regression, based on a theoretical expression that describes the decay of an autocorrelation function, $G(\tau)$, in consequence of diffusion and fast, non-diffusive processes, such as excitation of fluorescent molecules to the triplet state [31-33]:

$$
\begin{aligned}
G(\tau)= & \frac{\sum_{i} E_{i}^{2}\left\langle N_{i}\right\rangle M_{i}(\tau)}{\left(\sum_{i} E_{i}\left\langle N_{i}\right\rangle\right)^{2}} \cdot\left(1-F+F \cdot \exp \left(-\tau / \tau_{f}\right)\right) \\
& +G(\infty)
\end{aligned}
$$

Here, $E_{i}$ is the relative emission rate of species $i$, and $\left\langle N_{i}\right\rangle$ the average number of molecules of species $i$ in the focal volume. $M_{i}(\tau)$ is the mobility term for free, 3-dimensional diffusion:

$M_{i}(\tau)=\left(1+\tau / \tau_{D, i}\right)^{-1} \cdot\left(1+\left(r_{0}^{*} / z_{0}\right)^{2} \cdot\left(\tau / \tau_{D, i}\right)\right)^{-1 / 2}$ where $\tau_{D, i}$ is the diffusion time of fluorescent species $i$. The coefficients $r_{0}^{*}$ and $z_{0}$ are defined, respectively, as the distances from and along the optical axis at which the excitation light intensity has dropped by $1 / e^{2}$. These coefficients were determined from calibration of the focal volume with rhodamine green of known diffusion coefficient $\left(2.8 \times 10^{-6}\right.$ $\left.\mathrm{cm}^{2} \mathrm{~s}^{-1}\right)$.

The coefficient $F$ in Eq. (4) represents the fraction of molecules involved in a non-diffusive process, and $\tau_{f}$ is the decay time of this process. It is justified to assume the existence of a non-diffusive decay process, because the transition of excited state Nile red molecules to a non-emissive TICT state and to the triplet state has been proposed [19]. $G(\infty)$ is the value of the autocorrelation function at long time intervals $(\tau)$.

To reduce the number of fitting parameters, Eq. (4) was simplified. For one diffusing species, it can readily be written as:

$G(\tau)=\frac{1}{\langle N\rangle} \cdot M(\tau) \cdot\left(1-F+F \cdot \exp \left(-\tau / \tau_{f}\right)\right)+G(\infty)$

The assumption of two or more diffusing species gives:

$G(\tau)=\left(\sum_{i} f_{i} \cdot M_{i}(\tau)\right) \cdot\left(1-F+F \cdot \exp \left(-\tau / \tau_{f}\right)\right)+G(\infty)$

where

$f_{i}=E_{i}^{2}\left\langle N_{i}\right\rangle /\left(\sum_{i} E_{i}\left\langle N_{i}\right\rangle\right)^{2}$

Before data analysis, the experimental autocorrelation functions were truncated at very short time intervals below $0.001 \mathrm{~ms}$ and at very long time intervals above $600-1000 \mathrm{~ms}$. The autocorrelation functions were then normalized [34]:

$G_{\text {norm }}(\tau)=\frac{G(\tau)-G(\infty)}{G(0)-G(\infty)}$

where $G(0)$ is the start value of the autocorrelation function and $G(\infty)$ is its value at very long time intervals. $G(0)$ and $G(\infty)$ were estimated individually for every experimental autocorrelation function by non-linear regression, using Eqs. (6) and (7) [34]. Normalization eliminated possible differences in experimental autocorrelation functions that were related to systematic errors, such as adsorption of dye molecules to the surfaces of the well plate during experiments.

The above described normalization changes Eq. (6) to:

$$
\begin{aligned}
G_{\text {norm }}(\tau)= & G_{\text {norm }}(0) \cdot M(\tau) \cdot\left(1-F+F \cdot \exp \left(-\tau / \tau_{f}\right)\right) \\
& +G_{\text {norm }}(\infty)
\end{aligned}
$$


Here, $G_{\text {norm }}(0)$ is the start value of the normalized autocorrelation function, which is expected to be one. The value at long time intervals, accounted for by $G_{\text {norm }}(\infty)$, is expected to be zero. Equation (7) changes to:

$$
\begin{aligned}
G_{\text {norm }}(\tau)= & \left(\sum_{i} f_{i, \text { norm }} \cdot M_{i}(\tau)\right) \\
& \cdot\left(1-F+F \cdot \exp \left(-\tau / \tau_{f}\right)\right)+G_{\text {norm }}(\infty)
\end{aligned}
$$

where

$$
\begin{aligned}
f_{i, \text { norm }} & =\frac{E_{i}^{2}\left\langle N_{i}\right\rangle /\left(\sum_{i} E_{i}\left\langle N_{i}\right\rangle\right)^{2}}{\left(\sum_{i} E_{i}^{2}\left\langle N_{i}\right\rangle\right) /\left(\sum_{i} E_{i}\left\langle N_{i}\right\rangle\right)^{2}} \\
& =E_{i}^{2}\left\langle N_{i}\right\rangle /\left(\sum_{i} E_{i}^{2}\left\langle N_{i}\right\rangle\right)
\end{aligned}
$$

Equation (12) shows that $f_{i, \text { norm }}$ represents an intensityweighted fraction of diffusing species $i$. Equations (10) and (11) were used for analyzing the normalized, experimental autocorrelation functions by non-linear regression using the Levenberg-Marquardt algorithm. The calculations were performed with IGOR Pro V.4.04 (WaveMetrics, Inc., Lake Oswego, OR). When applicable, up to ten autocorrelation functions obtained from the same sample were concatenated into one data set and analyzed by a global analysis procedure contained in the software. The standard deviation $(\sigma)$ of experimental autocorrelation functions, estimated from the measurement repeats, was used as the weighting factor during fitting [34].

Solutions with a heterogeneous distribution of particle sizes showed very complex decays of the experimental autocorrelation functions. These could not be adequately fit by the discrete multi-exponential model using a small number of diffusion times, and increasing the number of diffusion times above three in the model resulted in substantially increased error margins of the determined parameters. For such complex data, calculation of the distribution of diffusion times was performed by the maximum entropy method (MEM) using the MEMFCS software [35].

Translation of diffusion time $\left(\tau_{D}\right)$ to a diffusion coefficient (D) was done by the following relationship:

$D=\left(r_{0}^{*}\right)^{2} / 4 \tau_{D}$

Calculation of the hydrodynamic radius $\left(R_{h}\right)$ was then possible using the Stokes-Einstein equation:

$D=k T / 6 \pi \cdot \eta \cdot R_{h}$

\section{Light scattering}

Detection of $\beta$-galactosidase aggregates in solution was done with static light scattering measurements using a fluorometer (Fluorolog FL3-21, Jobin Yvon-Horiba, Edison, NJ). The excitation and emission wavelengths were set to $400 \mathrm{~nm}$, and measurements were performed at $25^{\circ} \mathrm{C}$.

Furthermore, the hydrodynamic radius of native $\beta$ galactosidase was determined by dynamic light scattering measurements of $0.10 \mu \mathrm{M}$ protein solutions in phosphate buffer. Experiments were performed at $25^{\circ} \mathrm{C}$ with a CGS3 goniometer system and an LSE-5003 correlator (ALV, Langen, Germany). The average hydrodynamic radius $\left(R_{h}\right)$ of the $\beta$-galactosidase molecules was determined using the method of cumulants.

\section{Size exclusion chromatography (SEC)}

SEC measurements were performed using an Alliance 2695 system with a type 2487 dual wavelength detector and a type 2475 fluorescence detector (Waters, Milford, MA). A Superose 6 column with a separation range of $5 \times 10^{3}$ to $5 \times 10^{6} \mathrm{Da}$, and an exclusion limit of $4 \times 10^{7} \mathrm{Da}(\mathrm{GE}$ Healthcare Europe GmBH, Roosendaal, The Netherlands) was used. Phosphate buffered saline ( $\mathrm{pH}$ 7.4) was used as an eluent, and the injection volume was $40 \mu \mathrm{l}$. Detection was by UV absorption at $210 \mathrm{~nm}$ or by fluorescence emission $\left(\lambda_{\text {ex }} 280 \mathrm{~nm}\right.$ and $\lambda_{\text {em }} 340 \mathrm{~nm}$ for intrinsic protein fluorescence; $\lambda_{\text {ex }} 550 \mathrm{~nm}$ and $\lambda_{\mathrm{em}} 611 \mathrm{~nm}$ for Nile red fluorescence). Before injection, samples were centrifuged at 10000 RPM for 5 min. A molecular weight calibration curve using chymotrypsin $(25 \mathrm{kDa})$, human serum albumin (66 kDa and $132 \mathrm{kDa}$ dimer), and thyroglobulin $(670 \mathrm{kDa})$ as molecular weight standards was established.

\section{Results and discussion}

Heat denaturation of $\beta$-galactosidase

The temperature induced denaturation of $\beta$-galactosidase was followed by measuring intrinsic tryptophan fluorescence emission of this protein (not shown). Denaturation started between 50 and $55^{\circ} \mathrm{C}$ and was completed between 60 and $65^{\circ} \mathrm{C}$. An unfolding temperature of $57.4^{\circ} \mathrm{C}$ was obtained by fitting the denaturation curve with a model for a two-state unfolding process [36]. This is in good agreement with DSC experiments and activity assays performed under comparable conditions [25].

Heating $\beta$-galactosidase solutions above the unfolding temperature leads to irreversible aggregation of protein molecules [25]. Accordingly, a $0.10 \mu \mathrm{M} \beta$-galactosidase solution incubated at $62^{\circ} \mathrm{C}$ for $5 \mathrm{~min}$ was heavily aggregated 
and remained so upon cooling back to room temperature, as detected by light scattering (not shown). The aggregates formed under these conditions were too large to be detected by SEC. This is shown in Fig. 1a, which depicts SEC chromatograms of a non-heated $\beta$-galactosidase solution and of a $\beta$-galactosidase solutions that was heated at $62^{\circ} \mathrm{C}$ for $5 \mathrm{~min}$. The former consists of peaks representing native, tetrameric $\beta$-galactosidase, oligomers of two and three $\beta$-galactosidase molecules, and even some aggregates of higher molecular weight. The latter consists only of a peak representing native, tetrameric $\beta$-galactosidase. However, the area under the curve of this peak is reduced to about $10 \%$ of the area under the curve of the corresponding peak of non-heated $\beta$-galactosidase solution. The apparent loss of protein was caused by the formation of large aggregates that were excluded from the SEC column.

Static light scattering measurements using a fluorometer indicated that heating $\beta$-galactosidase solutions at $49^{\circ} \mathrm{C}$ also led to the formation of aggregates. Figure $1 \mathrm{~b}$ shows the intensity of scattered light of $0.10 \mu \mathrm{M} \beta$-galactosidase solutions heated for different times between $15 \mathrm{~min}$ and $6 \mathrm{~h}$. The increase of scattered light intensity indicates the formation of protein aggregates. This is clearly an effect of heating, because scattered light intensity did not increase upon storage at $25^{\circ} \mathrm{C}$ (not shown). Figure $1 \mathrm{~b}$ also shows that a maximum of scattered light intensity was reached after about $120 \mathrm{~min}$ of incubation. This is possibly related to the sedimentation of very large aggregates that had been formed after long incubation times, which would also explain the increasing error margins of the long time-points.

The SEC chromatograms in Fig. 1c show the formation of large $\beta$-galactosidase aggregates by heating solutions at $49^{\circ} \mathrm{C}$. Non-heated solution mainly contained the native, tetrameric $\beta$-galactosidase, along with some dimer, trimer, and even higher order aggregates. Heating for $15 \mathrm{~min}$ at $49^{\circ} \mathrm{C}$ did not lead to a change of the SEC chromatogram. However, after 60 min incubation an additional peak with a retention time of 29 min appeared, representing large $\beta$-galactosidase aggregates. The area of this peak was $1.5 \%$ of the total area under the curve of the protein and increased with increasing incubation time at $49^{\circ} \mathrm{C}$ (not shown). The chromatograms shown in Fig. 1c were obtained by measuring absorbance at $210 \mathrm{~nm}$. The use of an on-line fluorescence detector system $\left(\lambda_{\mathrm{ex}} 280 \mathrm{~nm}\right.$ and $\lambda_{\mathrm{em}} 340 \mathrm{~nm}$ ) led to corresponding results, i.e. aggregation was only detected in $\beta$-galactosidase solutions that were heated at $49^{\circ} \mathrm{C}$ for at least $60 \mathrm{~min}$.

Nile red steady-state fluorescence

Figure 2a depicts the emission spectra of Nile red in buffer, in non-heated $0.10 \mu \mathrm{M} \beta$-galactosidase solution, and in $0.10 \mu \mathrm{M} \beta$-galactosidase solution that was previously heated at $62^{\circ} \mathrm{C}$ for $5 \mathrm{~min}$. The emission of Nile red in the presence
(1)

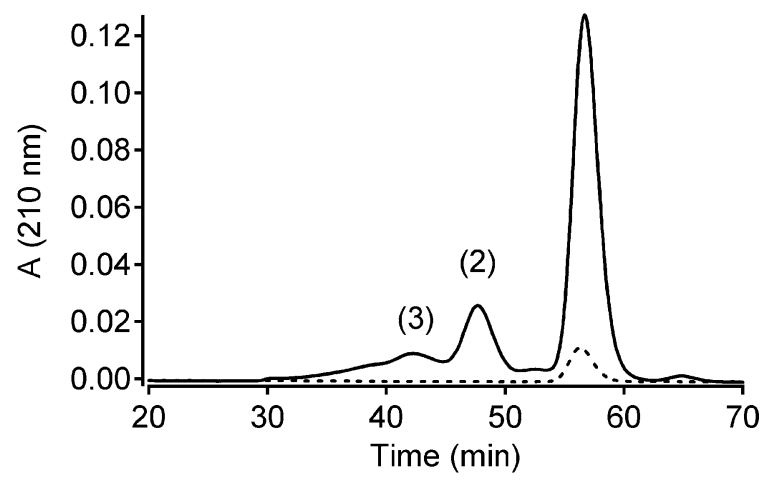

(a)

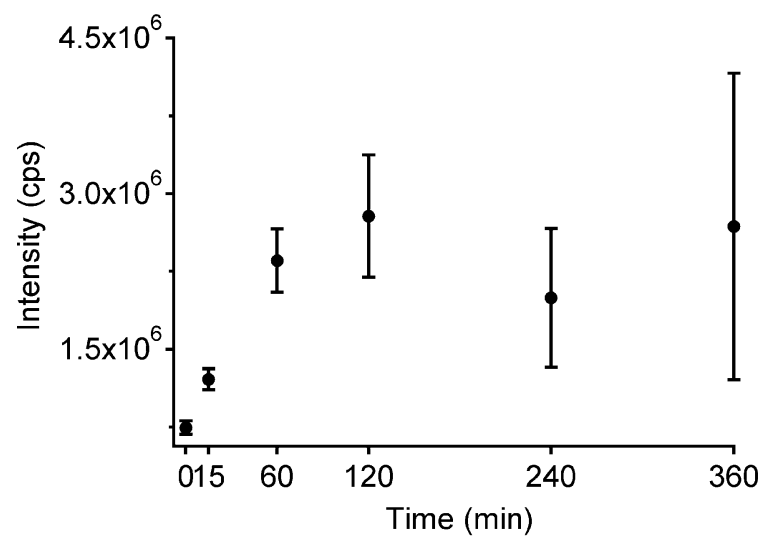

(b)

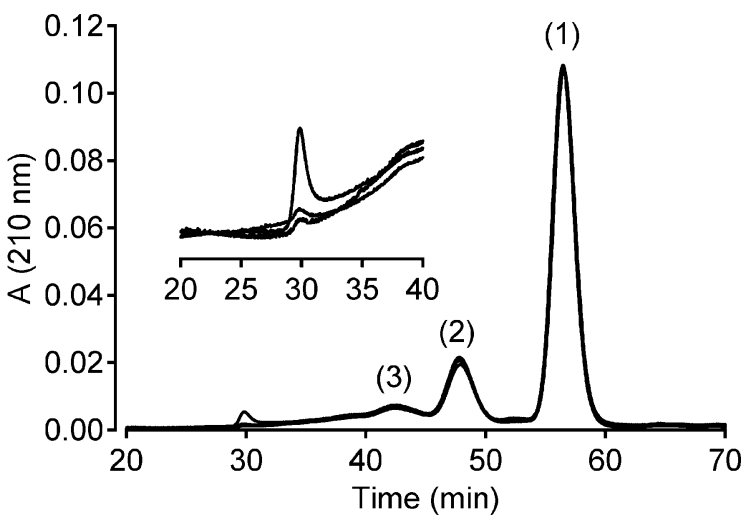

(c)

Fig. 1 (a) SEC chromatograms of non-heated $0.10 \mu \mathrm{M} \beta$ galactosidase solution (solid line) and $\beta$-galactosidase solution incubated at $62^{\circ} \mathrm{C}$ for $5 \mathrm{~min}$ (dotted line). The peaks represent native (i.e. tetrameric) $\beta$-galactosidase (1) and oligomers of two (2) and three (3) $\beta$-galactosidase molecules. (b) Plot of the increase of scattered light intensity at $400 \mathrm{~nm}$ against incubation time of $0.10 \mu \mathrm{M} \beta$-galactosidase solution at $49^{\circ} \mathrm{C}$. Data points are averages of 5-10 individual samples, and the error bars indicate \pm 1 SD. (c) SEC chromatograms of non-heated $0.10 \mu \mathrm{M} \beta$-galactosidase solution and solutions heated at $49^{\circ} \mathrm{C}$ for 15 and $60 \mathrm{~min}$. The peaks represent native, tetrameric $\beta$ galactosidase (1) and oligomers of two (2) and three (3) $\beta$-galactosidase molecules. The insert shows that after $60 \mathrm{~min}$ incubation, high-order aggregates of $\beta$-galactosidase appeared, with a retention time of $29 \mathrm{~min}$. There was no detectable difference between non-heated solutions and solutions heated at $49^{\circ} \mathrm{C}$ for $15 \mathrm{~min}$ 


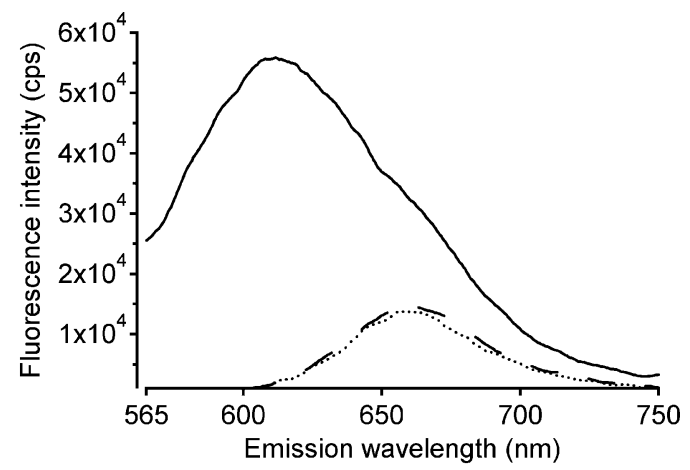

(a)


(b)

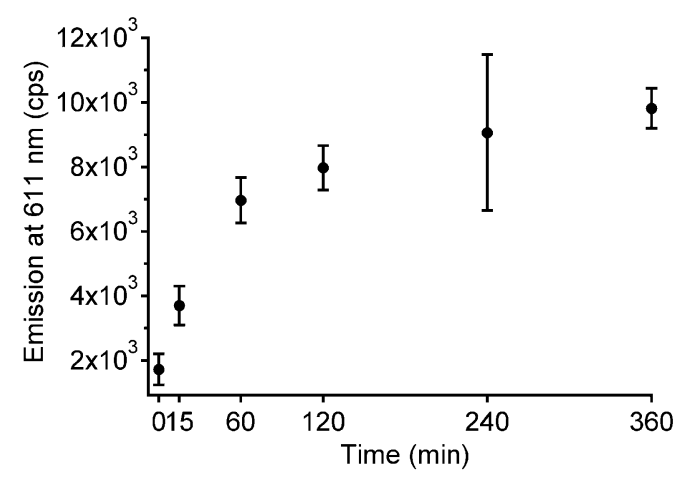

(c)

Fig. 2 (a) Fluorescence emission spectra of Nile red added to phosphate buffer (broken line; $\lambda_{\max }=662 \mathrm{~nm}$ ), non-heated $0.10 \mu \mathrm{M} \beta$ galactosidase solution (dotted line; $\lambda_{\max }=659 \mathrm{~nm}$ ), and $0.1 \mu \mathrm{M}$ $\beta$-galactosidase solution that was previously heated at $62^{\circ} \mathrm{C}$ for $5 \mathrm{~min}$ (solid line; $\lambda_{\max }=611 \mathrm{~nm}$ ). Excitation wavelength was $550 \mathrm{~nm}$. (b) Fit of two Voigt-shaped peaks (broken lines) to an emission spectrum (circles) of Nile red added to $\beta$-galactosidase solution that was heated at $49^{\circ} \mathrm{C}$ for $360 \mathrm{~min}$. The solid black line is the fitted spectrum, and the residuals are indicated. The positions of the fitted peaks are $605.0 \pm 2.2 \mathrm{~nm}$ and $658.3 \pm 1.4 \mathrm{~nm}$. (c) Plot of Nile red fluorescence intensity at $611 \mathrm{~nm}$ against incubation time at $49^{\circ} \mathrm{C}$ of $0.10 \mu \mathrm{M}$ $\beta$-galactosidase solution. Data points are averages of 5 individual samples, and the error bars indicate $\pm 1 \mathrm{SD}$ of non-heated protein was almost identical to the dye's emission in buffer. This indicates that the surface area of native $\beta$-galactosidase is highly polar, and that possible apolar sites are not accessible to Nile red. Storage of unheated $\beta$ galactosidase solutions containing Nile red for up to $25 \mathrm{~min}$ at room temperature did not lead to a change of the dyes fluorescence, showing that the presence of Nile red does not lead to a change of $\beta$-galactosidase surface hydrophobicity over time.

Interaction of Nile red with heat-denatured $\beta$ galactosidase resulted in a shift of emission maximum from 660 to $611 \mathrm{~nm}$ and in a substantial increase in fluorescence intensity (Fig. 2a). This is typically observed when Nile red molecules bind to hydrophobic, apolar sites on the surface of a protein [14], and shows that irreversible denaturation and aggregation by heating above the unfolding temperature has increased the surface hydrophobicity of $\beta$-galactosidase.

Upon 15 min incubation of $0.10 \mu \mathrm{M} \beta$-galactosidase solution at $49^{\circ} \mathrm{C}$, the emission spectrum of Nile red reproducibly showed an increased intensity below $640 \mathrm{~nm}$. Upon longer incubation between 1 and $6 \mathrm{~h}$, a shoulder between 605 and $615 \mathrm{~nm}$ emerged. Figure 2b shows a two-peak fit of an emission spectrum obtained with Nile red added to $\beta$ galactosidase solution that was previously heated at $49^{\circ} \mathrm{C}$ for $6 \mathrm{~h}$. In Fig. 2b, the $\lambda_{\text {max }}$ of the main peak is $658 \mathrm{~nm}$, whereas the peak responsible for the shoulder is positioned at $605 \mathrm{~nm}$. Emission at $658 \mathrm{~nm}$ originates from free Nile red molecules, and emission at $605 \mathrm{~nm}$ from Nile red interacting with denatured $\beta$-galactosidase. The latter is comparable with the $\lambda_{\max }$ of Nile red in $\beta$-galactosidase solution that had been heated above the unfolding temperature (Fig. 2a). This indicates that the surface polarity of protein molecules denatured at different temperatures is similar. The fluorescence of Nile red did not depend on the time that protein solutions were kept at room temperature upon heating at $49^{\circ} \mathrm{C}$ (not shown), which indicates that irreversibly denatured $\beta$-galactosidase species were detected.

The effect of increasing incubation time at $49^{\circ} \mathrm{C}$ on Nile red fluorescence intensity is shown in Fig. 2c. An observation wavelength of $611 \mathrm{~nm}$ was chosen, which corresponds to the average emission wavelength of Nile red interacting with irreversibly denatured $\beta$-galactosidase. The profile of Fig. $2 c$ is similar to that of Fig. 1b, suggesting that Nile red interacted with aggregated $\beta$-galactosidase. A clear decrease of Nile red emission at $611 \mathrm{~nm}$ upon centrifugation at 10000 RPM (not shown) supported this notion. However, it was not possible to confirm this by SEC, neither by adding Nile red to the samples before injection and performing online fluorescence detection $\left(\lambda_{\text {ex }} 550 \mathrm{~nm}\right.$ and $\lambda_{\text {em }} 611 \mathrm{~nm}$ ), nor by staining fractions from SEC runs with Nile red and performing off-line fluorescence detection. For this reason, time-resolved fluorescence spectroscopy and FCS were used 


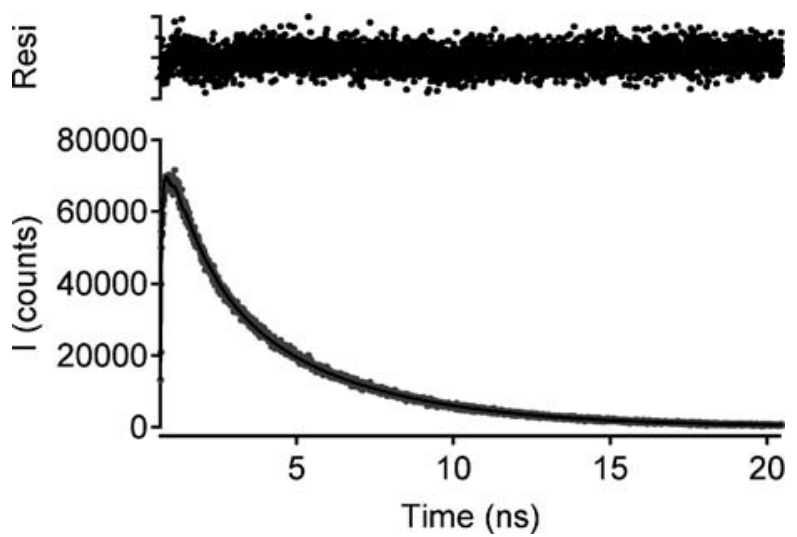

Fig. 3 Fluorescence intensity decay, $I(t)$, of Nile red in $0.10 \mu \mathrm{M} \beta$ galactosidase solution that was heated at $49^{\circ} \mathrm{C}$ for $60 \mathrm{~min}$. The fitted decay curve and the weighted residuals are shown. Fitting gave three lifetimes $\left(\tau_{1}=0.11, \tau_{2}=1.11\right.$, and $\tau_{3}=4.36 \mathrm{~ns}$ ) and three (normalized) pre-exponential factors $\left(\alpha_{1, \text { norm }}=0.40, \alpha_{2, \text { norm }}=0.24\right.$, and $\left.\alpha_{3, \text { norm }}=0.36\right)$. The value of $\chi^{2}$ was 1.056

to further investigate the interaction between Nile red and aggregated $\beta$-galactosidase.

Time-resolved fluorescence spectroscopy with Nile red

Figure 3 shows the analysis of the fluorescence intensity decay, $I(t)$, of Nile red in $0.10 \mu \mathrm{M} \beta$-galactosidase solution that was heated at $49^{\circ} \mathrm{C}$ for $60 \mathrm{~min}$. The data points could be adequately described using Eq. (1) with three fluorescent lifetimes, as can be seen from the regular distribution of the residuals around zero and the proximity of $\chi^{2}$ to unity. The use of three lifetimes was necessary for the analysis of all intensity decays, and it was not possible to relate particular lifetimes to the free and the protein-bound state of Nile red. In Table 1 , the results are summarized by listing $\langle\tau\rangle$ values, which are proportional to steady state intensities. In agreement with the increase of Nile red steady state intensity at $611 \mathrm{~nm}$, heating of $\beta$-galactosidase solution at $49^{\circ} \mathrm{C}$ for $15 \mathrm{~min}$ led to a clear increase of $\langle\tau\rangle$ as compared to Nile red in buffer or non-heated protein solution. The tendency

Table 1 Lifetime analysis

\begin{tabular}{lll}
\hline Sample & $\chi^{2}$ total $^{a}$ & $\langle\tau\rangle^{b}$ \\
\hline buffer & 1.039 & 0.20 \\
non-heated & 1.011 & 0.42 \\
$15-49^{\circ} \mathrm{C}$ & 1.062 & 1.30 \\
$60-49^{\circ} \mathrm{C}$ & 1.056 & 1.89 \\
$120-49^{\circ} \mathrm{C}$ & 1.064 & 2.48 \\
$240-49^{\circ} \mathrm{C}$ & 1.000 & 2.10 \\
$5-62^{\circ} \mathrm{C}$ & 1.032 & 1.50 \\
\hline${ }^{a} \chi^{2}$ obtained with Eq. $(1)$ using three fluorescence \\
lifetimes. \\
${ }^{b}\langle\tau\rangle=\sum \alpha_{i, \text { norm }} \cdot \tau_{i}$.
\end{tabular}

of $\langle\tau\rangle$ to further increase with increasing heating time at $49^{\circ} \mathrm{C}$ corresponds nicely to the increase of fluorescence intensity upon prolonged heating shown in Fig. 2c. Also with $\beta$-galactosidase solution that was heated at $62^{\circ} \mathrm{C}$ for $5 \mathrm{~min}$, $\langle\tau\rangle$ was substantially increased as compared to non-heated protein solution. However, it was lower than expected from the steady-state fluorescence intensity at $611 \mathrm{~nm}$ (Fig. 2a). Possible reasons for this difference are the variation between individual experiments with solutions containing highly aggregated protein and, since there is a lot of light scattering in such samples, different sensitivities of the steady-state and time-resolved instrumentation for scattered light.

The course of fluorescence anisotropy with time, $r(t)$, of Nile red in $0.10 \mu \mathrm{M} \beta$-galactosidase solution that was heated at $49^{\circ} \mathrm{C}$ for $15,60,120$, and $240 \mathrm{~min}$ was measured (not shown). With all samples, anisotropy decreased at short times, then increased again, and finally remained at an almost constant value. The practically constant value of anisotropy at long times indicated rotational diffusion that was too slow to be detected by Nile red fluorescence. Therefore, although indicating that Nile red was in fact bound to large particles such as aggregates of $\beta$-galactosidase, it was not possible to estimate the size of these particles by time-resolved fluorescence anisotropy.

\section{Fluorescence correlation spectroscopy (FCS)} measurements with Nile red

Figure 4a depicts an overlay of ten normalized, experimental autocorrelation functions that were obtained with Nile red in buffer. These data were analyzed simultaneously using the one-component model (Eq. (10)). The fitted curve included in Fig. $4 \mathrm{a}$, the regular distribution of the weighted residuals around zero, and the proximity of $\chi^{2}$ to unity show that Eq. (10) was appropriate for describing the experimental data. The determined decay time for non-diffusive process $\left(\tau_{f}\right)$ was $0.022 \mathrm{~ms}$, the fraction of dye molecules involved in this process $(F)$ was 0.45 , and the determined diffusion time $\left(\tau_{D, 1}\right)$ was $0.309 \mathrm{~ms}$ (Table 2$)$. A diffusion time of Nile red around $0.3 \mathrm{~ms}$ is in agreement with reported values [37]. It corresponds to a diffusion coefficient of $2.9 \times 10^{-6} \mathrm{~cm}^{2} \mathrm{~s}^{-1}$, which is reasonable for a molecule with a molecular weight of $318 \mathrm{Da}$. Therefore, $\tau_{D, 1}$ can be associated with the diffusion of free dye molecules. $G_{\text {norm }}(0)$ deviated slightly from the expected value of unity (Table 2). Forcing it to unity increased $\chi^{2}$ slightly, but had no effect on the determined parameters $\tau_{D, 1}, \tau_{f}$, and $F$. Also $G_{\text {norm }}(\infty)$ deviated somewhat from the expected value of zero (Table 2). This is related to the background subtraction described in the materials and methods section. Leaving out background subtraction did not lead to different values of the coefficients $\tau_{D, 1}, \tau_{f}$, and $F$. Measurement of Nile red in non-heated $0.10 \mu \mathrm{M} \beta$-galactosidase solution gave the same parameters 


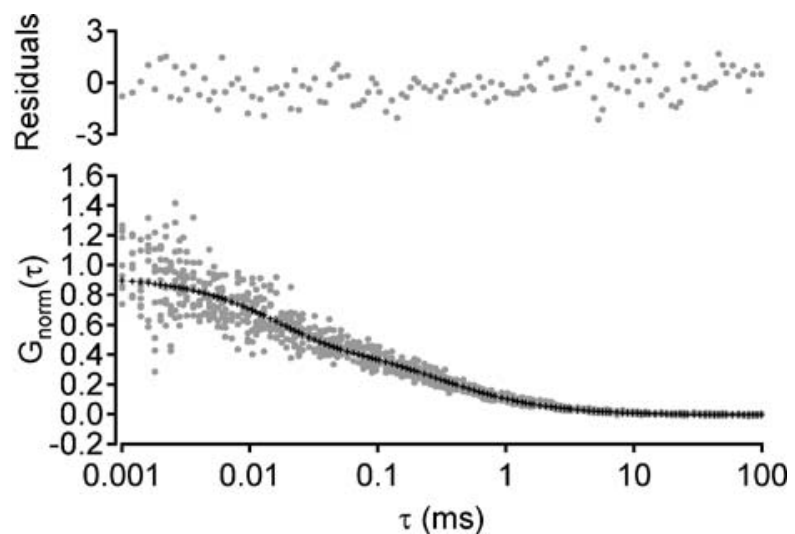

(a)
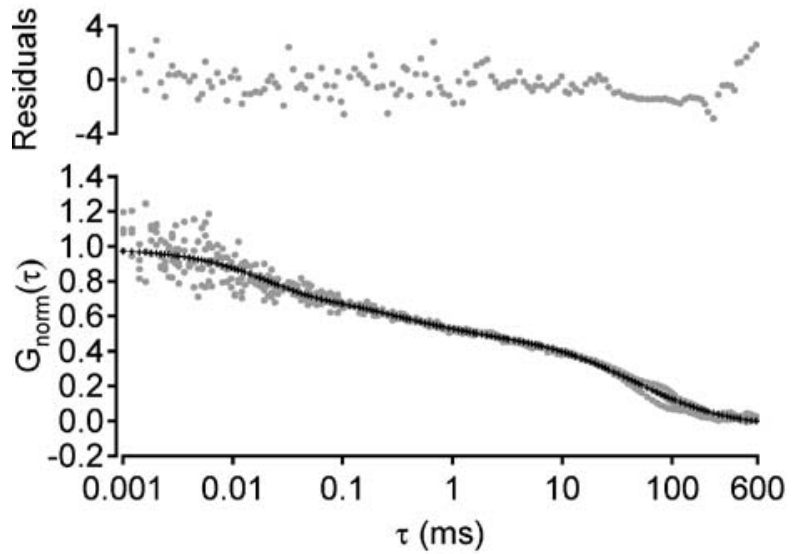

(b)

Fig. 4 Analysis of FCS data. (a) Simultaneous fit of 10 normalized autocorrelation functions obtained with Nile red in buffer. Analysis was performed by non-linear regression using Eq. (10). The fitted curve (black) and the weighted residuals for one of the normalized autocorrelation functions are shown. Determined parameters (value \pm 1 SD) were $F=0.451 \pm 0.010, \tau_{\mathrm{f}}=0.022 \pm 0.002 \mathrm{~ms}, \tau_{D, 1}=0.309 \pm 0.008$ $\mathrm{ms}, G_{\text {norm }}(0)=0.920 \pm 0.016$, and $G_{\text {norm }}(\infty)=-0.0049 \pm 0.0003$. The value of $\chi_{r}^{2}$ was 1.03. (b) Simultaneous fit of 9 normalized autocorrelation functions obtained with Nile red in $0.10 \mu \mathrm{M} \beta$-galactosidase solution that was previously heated at $62^{\circ} \mathrm{C}$ for $5 \mathrm{~min}$. Analysis was performed by non-linear regression using Eq. (11) for two diffusing species. The fitted curve (black) and the weighted residuals for one of the normalized autocorrelation functions are shown. Determined parameters (value $\pm 1 \mathrm{SD}$ ) were $F=0.246 \pm 0.008, \tau_{\mathrm{f}}=0.019 \pm$ $0.001 \mathrm{~ms}, \mathrm{f}_{1, \text { norm }}=0.335 \pm 0.005, \tau_{D, 1}=0.287 \pm 0.017 \mathrm{~ms}, f_{2, \text { norm }}=$ $0.690 \pm 0.007, \tau_{D, 2}=45.70 \pm 1.02 \mathrm{~ms}$, and $G_{\text {norm }}(\infty)=-0.037 \pm$ 0.0021 . The value of $\chi_{r}^{2}$ was 1.44

as Nile red in buffer (Table 2), confirming that there was no substantial interaction between the dye and non-heated protein.

An overlay of nine normalized, experimental autocorrelation functions, obtained with Nile red in $0.10 \mu \mathrm{M} \beta$ galactosidase solution that was previously heated at $62^{\circ} \mathrm{C}$ for $5 \mathrm{~min}$, is shown in Fig. 4b. There is an additional decay of the autocorrelation functions between 20 and $200 \mathrm{~ms}$, which was not observed with Nile red in buffer. Data analysis was therefore performed using a two-component model,

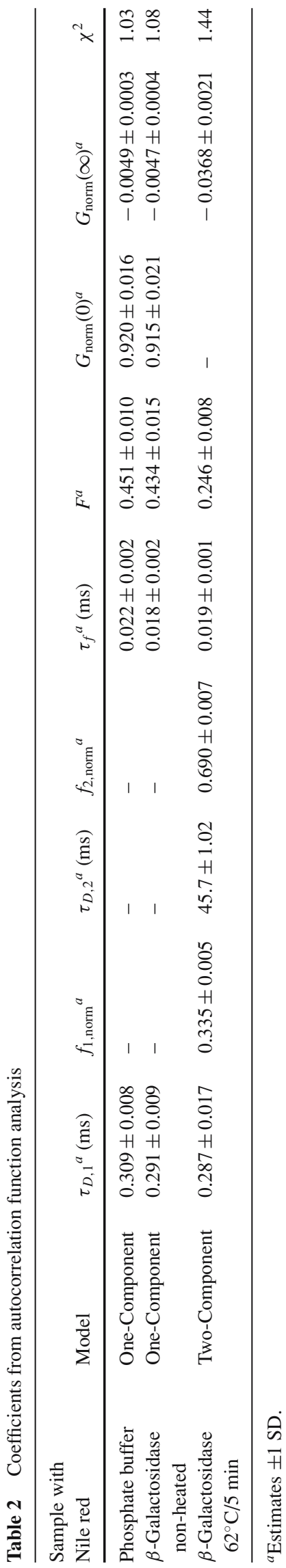


i.e. Eq. (11) assuming two diffusing species. The fitted line and the weighted residuals in Fig. $4 \mathrm{~b}$ show that up to time intervals between 1 and $10 \mathrm{~ms}$, the data could be well described by the two component model. Within this range, the decay of the autocorrelation functions is governed by $\tau_{f}$ and $\tau_{D, 1}$. The determined values were 0.019 and $0.287 \mathrm{~ms}$, respectively (Table 2), which is in good agreement with the values found for Nile red in buffer. This shows that also in the heated $\beta$-galactosidase solution, not all Nile red was bound to denatured protein. The fraction of dye molecules $(F)$ involved in non-diffusive process was 0.25 , which is substantially lower than for Nile red in buffer (Table 2). A possible explanation for this is that in solutions with large amounts of denatured protein, less Nile red molecules undergo transition to the TICT and triplet state, because they can interact with hydrophobic surfaces [19]. The second diffusion time $\left(\tau_{D, 2}\right)$ of $46 \mathrm{~ms}$ (Table 2$)$ governed the decay of autocorrelation functions between 20 and $200 \mathrm{~ms}$. It corresponds to a diffusion coefficient $(D)$ of $1.9 \times 10^{-8} \mathrm{~cm}^{2} \mathrm{~s}^{-1}$

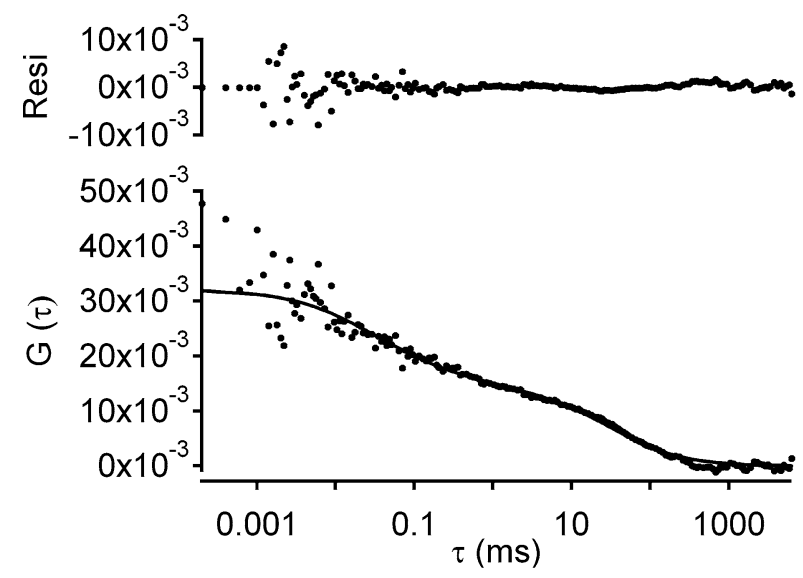

(a)

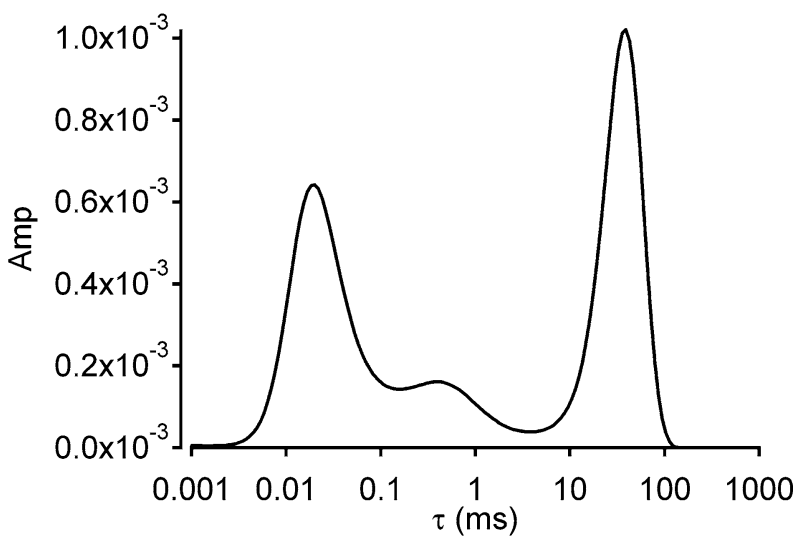

(b)

Fig. 5 Analysis of FCS data. (a) MEM analysis of a single autocorrelation function obtained with Nile red in $0.10 \mu \mathrm{M} \beta$-galactosidase solution that was previously heated at $62^{\circ} \mathrm{C}$ for $5 \mathrm{~min}$. The non-weighted residuals are shown. (b) Distribution of diffusion times $\left(\tau_{D}\right)$ calculated by MEM analysis and a hydrodynamic radius $\left(R_{h}\right)$ of $130 \mathrm{~nm}$, which deviates substantially from the values of native $\beta$-galactosidase $\left(D=3.1 \times 10^{-7} \mathrm{~cm}^{2} \mathrm{~s}^{-1} ; R_{h}=8 \mathrm{~nm}\right)$. This shows that incubation at $62^{\circ} \mathrm{C}$ for $5 \mathrm{~min}$ had led to very large, denatured aggregates of $\beta$-galactosidase, which could be detected by Nile red. However, the two-component model (Eq. (11)) was not able to fully describe the very complex autocorrelation function decays shown in Fig. $4 \mathrm{~b}$, as reflected by the residuals and the relatively high $\chi^{2}$ value of 1.44 (Table 2). For this reason, the distribution of diffusion times was determined by the maximum entropy method (MEM) [35]. Such an analysis is shown in Fig. 5a for an individual experimental autocorrelation function. The distribution of diffusion times (Fig. 5b) shows 3 peaks, centered at $0.02,0.36$, and $39 \mathrm{~ms}$. The 0.02 and $0.36 \mathrm{~ms}$ peaks correspond to $\tau_{f}$ and $\tau_{D, 1}$ determined with Eq. (11). The peak around $39 \mathrm{~ms}$ is very broad and ranges from 5 to $150 \mathrm{~ms}$, which corresponds to hydrodynamic radii between 14 and $420 \mathrm{~nm}$. The peak maximum of $39 \mathrm{~ms}$ corresponds to an $R_{h}$ of $109 \mathrm{~nm}$, in good agreement with $\tau_{D, 2}$ obtained with Eq. (11). This is well above the hydrodynamic radius of small products of $\beta$-galactosidase denaturation, i.e. denatured monomers, dimers, and tetramers [25], and confirms that interaction with large $\beta$-galactosidase aggregates was the reason for the change of Nile red fluorescence.

Nile red also detected large aggregates in $0.10 \mu \mathrm{M} \beta$ galactosidase solutions that had been incubated at $49^{\circ} \mathrm{C}$. As for protein samples heated at $62^{\circ} \mathrm{C}$, these aggregates were detectable by an additional decrease of FCS autocorrelation functions at long times. However, aggregate concentration in samples heated at $49^{\circ} \mathrm{C}$ was much lower than in samples heated at $62^{\circ} \mathrm{C}$. This is shown in Fig. 6, which compares the raw FCS data obtained with Nile red in $\beta$-galactosidase solutions heated for $5 \mathrm{~min}$ at $62^{\circ} \mathrm{C}$ and for $15 \mathrm{~min}$ at $49^{\circ} \mathrm{C}$. The former is full of high-intensity peaks that were caused by large aggregates with bound Nile red crossing the focal

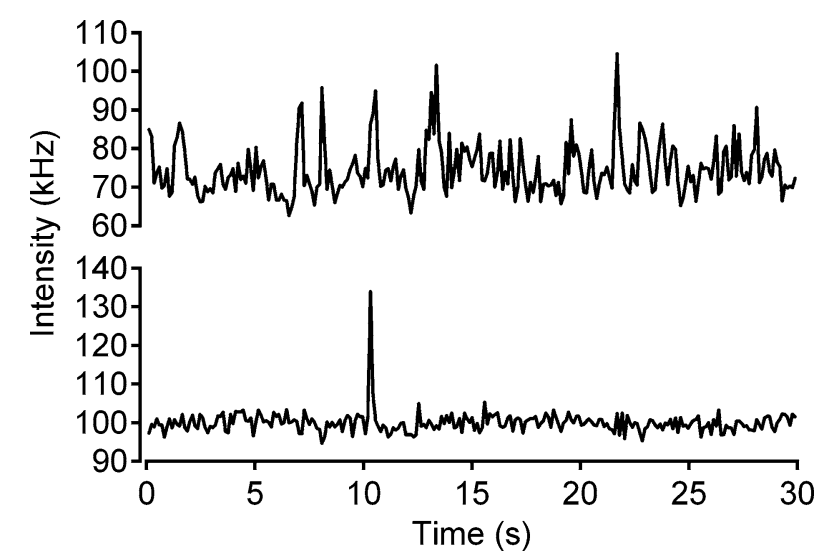

Fig. 6 Raw data of two FCS measurements obtained with $0.10 \mu \mathrm{M} \beta$ galactosidase solution that was heated at $62^{\circ} \mathrm{C}$ for $5 \min$ (upper curve) and at $49^{\circ} \mathrm{C}$ for $15 \mathrm{~min}$ 
volume. The latter contains only one high-intensity peak, representing a single large aggregate crossing the focal volume. As a consequence of the low aggregate concentration in samples heated at $49^{\circ} \mathrm{C}$, only 3 out of 100 measurements contained high-intensity peaks. In spite of their rare occurrence, these peaks were not artifacts. No high-intensity peaks were detected in a total of 150 control experiments with Nile red in buffer and in non-heated $\beta$-galactosidase solution.

The combined results of steady-state fluorescence spectroscopy, time-resolved fluorescence spectroscopy, and FCS show that Nile red fluorescence gave complementary information to SEC about the aggregation of $\beta$-galactosidase. Native protein molecules and small oligomers could be detected by SEC (Fig. 1a and c), but did not substantially affect Nile red fluorescence. It is possible that both the surface hydrophobicity and the surface area of these protein species were not sufficient for an effective interaction with the dye. However, Nile red was able to interact with large, denatured aggregates of $\beta$-galactosidase and could therefore be used as a probe for their specific detection. Although some higher order aggregates could be detected by SEC upon heating at $49^{\circ} \mathrm{C}$ (Fig. 1c), Nile red fluorescence was sensitive to the presence of even larger aggregates, which were missed by SEC (Fig. 1a).

The interaction of Nile red with large aggregates of $\beta$ galactosidase makes it very difficult to use the SEC results for estimating the sensitivity of the Nile red method. Figure 1c shows that some large aggregates were first detected by SEC in protein samples that had been heated at $49^{\circ} \mathrm{C}$ for $60 \mathrm{~min}$. However, the inability of Nile red to stain these aggregates fractionated from SEC runs (see results above) and the possible exclusion from the SEC column of even larger protein aggregates, which are detectable by Nile red, render the use of SEC as a reference method questionable. Furthermore, Nile red fluorescence was clearly more sensitive than SEC, as it already detected irreversible changes in samples that were heated at $49^{\circ} \mathrm{C}$ for $15 \mathrm{~min}$. Therefore, to estimate the sensitivity of Nile red, non-heated $\beta$-galactosidase solutions were doped with defined amounts of $\beta$-galactosidase solution that had been irreversibly denatured and aggregated by heating at $62^{\circ} \mathrm{C}$ for $5 \mathrm{~min}$. The lower limit of detection by Nile red steady-state fluorescence was $1 \%(\mathrm{v} / \mathrm{v})$ of heated protein solution, corresponding to $1 \mathrm{nM}$ denatured and aggregated $\beta$-galactosidase. The fluorescence intensity of this solution at $611 \mathrm{~nm}$ corresponded to that of $\beta$-galactosidase solution that had been heated at $49^{\circ} \mathrm{C}$ for 15 min (Fig. 2c). This comparison is justified, because nearly all $\beta$-galactosidase molecules had formed large aggregates upon heating at $62^{\circ} \mathrm{C}$ for $5 \mathrm{~min}$ (Fig. 1a), and because the fluorescence intensity of the detected aggregates did not depend on the incubation temperature (Fig. 6), which means that Nile red was equally sensitive to aggregates formed by heating at 49 and $62^{\circ} \mathrm{C}$.

\section{Conclusions}

Using the model protein $\beta$-galactosidase, it was shown that the presence small amounts of large, denatured protein aggregates in solution can be detected by Nile red fluorescence. Aggregates detected by Nile red had hydrodynamic radii around $130 \mathrm{~nm}$ with a broad size distribution. Native protein and small aggregates thereof had no substantial effect on Nile red fluorescence intensity. By steady-state fluorescence measurements, it was possible to detect $1 \mathrm{nM}$ denatured and highly aggregated $\beta$-galactosidase in solution.

The spectroscopic detection of protein aggregates by Nile red is potentially useful for formulation screening or quality control of protein pharmaceutics. The presence of even minute fractions of aggregates in protein formulations needs to be avoided, because this can cause immune reactions in patients. Since large aggregates are particularly potent for breaking immune tolerance, their analytical detection is very important. SEC, which is the standard method for studying protein aggregation, is not reliable for detecting large aggregates, because they may be excluded from the separation column. In this work we showed that an analytical detection method with Nile red is a possible approach to overcome this shortcoming of SEC. After establishing the method with time-resolved fluorescence spectroscopy and FCS, as was done for $\beta$-galactosidase, steady-state fluorescence measurements, which can be performed in most labs, may enable an experimentally simple and sensitive detection of large protein aggregates.

Acknowledgments We thank S. Maiti of the Tata Institute of Fundamental Research in Mumbai, India, for providing us with the MEMFCS software.The post-doctoral fellowship of N.N. Sanders is supported by the Fund for Scientific Research, Flanders, Belgium.

\section{References}

1. Walsh G (2004) Second-generation biopharmaceuticals. Eur J Pharm Biopharm 58:185-196

2. Hermeling S, Schellekens H, Crommelin DJ, Jiskoot W (2003) Micelle-associated protein in epoetin formulations: A risk factor for immunogenicity? Pharm Res 20:1903-1907

3. Hermeling S, Crommelin DJ, Schellekens H, Jiskoot W (2004) Structure-immunogenicity relationships of therapeutic proteins. Pharm Res 21:897-903

4. Hermeling S, Aranha L, Damen JM, Slijper M, Schellekens H, Crommelin DJA, Jiskoot W (2005) Structural characterization and immunogenicity in wild-type and immune tolerant mice of degraded recombinant human interferon alpha2b. Pharm Res 22:1997-2006

5. Philo JS (2005) Analytical Ultracentrifugation. In: Jiskoot W, Crommelin DJA (eds) Methods for structural analysis of protein pharmaceuticals, 1st edn. AAPS Press, Arlington, pp 379-412

6. Fraunhofer W, Winter G (2004) The use of asymmetrical flow fieldflow fractionation in pharmaceutics and biopharmaceutics. Eur $\mathbf{J}$ Pharm Biopharm 58:369-383 
7. Weijers M, Visschers RW, Nicolai T (2002) Light scattering study of heat-induced aggregation and gelation of ovalbumin. Macromolecules 35:4753-4762

8. Nielsen L, Frokjaer S, Carpenter JF, Brange J (2001) Studies of the structure of insulin fibrils by Fourier transform infrared (FTIR) spectroscopy and electron microscopy. J Pharm Sci 90:29-37

9. Bauer HH, Muller M, Goette J, Merkle HP, Fringeli UP (1994) Interfacial adsorption and aggregation associated changes in secondary structure of human calcitonin monitored by ATR-FTIR spectroscopy. Biochemistry 33:12276-12282

10. van de Weert M, Haris PI, Hennink WE, Crommelin DJA (2001) Fourier transform infrared spectrometric analysis of protein conformation: effect of sampling method and stress factors. Anal Biochem 297:160-169

11. Pitschke M, Prior R, Haupt M, Riesner D (1998) Detection of single amyloid beta-protein aggregates in the cerebrospinal fluid of Alzheimer's patients by fluorescence correlation spectroscopy. Nat Med 4:832-834

12. Puchtler H, Sweat F, Levine M (1962) On the binding of Congo red to amyloid. J Histochem Cytochem 10:355-364

13. Linke RP (2000) Highly sensitive diagnosis of amyloid and various amyloid syndromes using Congo red fluorescence. Virchows Arch 436:439-448

14. Sackett DL, Wolff J (1987) Nile red as a polarity-sensitive fluorescent probe of hydrophobic protein surfaces. Anal Biochem 167:228-234

15. Sackett DL, Knutson JR, Wolff J (1990) Hydrophobic surfaces of tubulin probed by time-resolved and steady-state fluorescence of Nile red. J Biol Chem 265:14899-14906

16. Kotik M, Zuber H (1992) Evidence for temperature-dependent conformational changes in the $L$-lactate dehydrogenase from Bacillus stearothermophilus. Biochemistry 31:7787-7795

17. Hendriks J, Gensch T, Hviid L, van der Horst MA, Hellingwerf KJ, van Thor JJ (2002) Transient exposure of hydrophobic surface in the photoactive yellow protein monitored with Nile red. Biophys J 82:1632-1643

18. Demeule B, Gurny R, Arvinte T (2006) Detection and characterization of protein aggregates by fluorescence microscopy. Int $\mathrm{J}$ Pharmaceutics (In press)

19. Sarkar N, Das K, Nath DN, Bhattacharyya K (1994) Twisted charge transfer process of Nile red in homogeneous solution and in faujasite zeolite. Langmuir 10:326-329

20. Dutta AK, Kamada K, Ohta K (1996) Spectroscopic studies of Nile red in organic solvents and polymers. J Photochem Photobiol A 93:57-64

21. Gautier S, Boustta M, Vert M (1999) Alkylated poly(L-lysine citramide) as models to investigate the ability of amphiphilic macromolecular drug carriers to physically entrap lipophilic compounds in aqueous media. J Control Release 60:235-247
22. Greenspan P, Fowler SD (1985) Spectrofluorometric studies of the lipid probe, Nile red. J Lipid Res 26:781-789

23. Huber RE, Parfett C, Woulfe-Flanagan H, Thompson DJ (1979) Interaction of divalent cations with $\beta$-galactosidase (Escherichia coli). Biochemistry 18:4090-4095

24. Juers DH, Heightman TD, Vasella A, McCarter JD, Mackenzie L, Withers SG, Matthews BW (2001) A structural view of the action of Escherichia coli (lacZ) $\beta$-galactosidase. Biochemistry 40:14781-14794

25. Edwards RA, Jacobson AL, Huber RE (1990) Thermal denaturation of $\beta$-galactosidase and of two site-specific mutants. Biochemistry 29:11001-11008

26. Edwards RA, Huber RE (1992) Surface denaturation of proteins: The thermal inactivation of $\beta$-galactosidase (Escherichia coli) on wall-liquid surfaces. Biochem Cell Biol 70:63-69

27. Borst JW, Hink MA, van Hoek A, Visser AJWG (2005) Effects of refractive index and viscosity on fluorescence and anisotropy decays of enhanced cyan and yellow fluorescent proteins. J Fluoresc $15: 153-160$

28. Digris AV, Skakun VV, Novikov EG, van Hoek A, Claiborne A, Visser AJWG (1999) Thermal stability of a flavoprotein assessed from associative analysis of polarized time-resolved fluorescence spectroscopy. Eur Biophys J 28:526-531

29. Jiskoot W, Visser AJWG, Herron JN, Sutter M (2005) Fluorescence spectroscopy. In: Jiskoot W, Crommelin DJA (eds) Methods for structural analysis of protein pharmaceuticals, 1st edn. AAPS Press, Arlington, pp 27-82

30. Lakowicz JR (1999) Principles of fluorescence spectroscopy, 2nd edn. Kluwer Academic/Plenum Publishers, New York

31. Meseth U, Wohland T, Rigler R, Vogel H (1999) Resolution in fluorescence correlation measurements. Biophys J 76:1619-1631

32. Bacia K, Majoul IV, Schwille P (2002) Probing the endocytic pathway in live cells using dual-color fluorescence cross-correlation analysis. Biophys J 83:1184-1193

33. Thompson NL (1991) Fluorescence Correlation Spectroscopy. In Lakowicz JR (ed) Topics in fluorescence spectroscopy, vol 1, Techniques, 1st edn. Plenum Press, New York, pp 337-378

34. Wohland T, Rigler R, Vogel H (2001) The standard deviation in fluorescence correlation spectroscopy. Biophys J 80:2987-2999

35. Sengupta P, Garai K, Balaji J, Periasamy N, Maiti S (2003) Measuring size distribution in highly heterogeneous systems with fluorescence correlation spectroscopy. Biophys J 84:19771984

36. Swint L, Robertson AD (1993) Thermodynamics of unfolding for turkey ovomucoid third domain: Thermal and chemical denaturation. Protein Sci 2:2037-2049

37. Sengupta P, Balaji J, Maiti S (2002) Measuring diffusion in cell membranes by fluorescence correlation spectroscopy. Methods $27: 374-387$ 